\title{
A Case of Harvesting Anterolateral Thigh Flaps Twice from the Ipsilateral Thigh of a Single Patient in Separate Operations
}

\author{
Saito Masami ${ }^{*}$, Kimura Naohiro², Okochi Masayuki' ${ }^{1}$, Tomizuka Yosuke ${ }^{3}$, \\ Onda Masamitsu ${ }^{4}$, Ueda Kazuki ${ }^{1}$ \\ ${ }^{1}$ Department of Plastic and Reconstructive Surgery, Fukushima Medical University, Fukushima, Japan \\ ${ }^{2}$ Tsukiji Dermatology Plastic and Hand Surgery, Tokyo, Japan \\ ${ }^{3}$ Department of Plastic and Reconstructive Surgery, Social Insurance Funabashi Central Hospital, Funabashi, \\ Japan \\ ${ }^{4}$ Shinwa Clinic Shinjuku, Tokyo, Japan \\ Email: *aamnnty0418@gmail.com
}

Received 2 March 2016; accepted 9 April 2016; published 12 April 2016

Copyright (C) 2016 by authors and Scientific Research Publishing Inc.

This work is licensed under the Creative Commons Attribution International License (CC BY).

http://creativecommons.org/licenses/by/4.0/

c) (i) Open Access

\begin{abstract}
Until now, harvesting of flap elevations from the ipsilateral thigh twice in the same patient in two operations has not been reported. We harvested thigh flaps twice from the ipsilateral thigh of a single patient in separate operations. A 44-year-old man had skin defects of the right thumb and the left middle finger. In the first operation, his right thumb was reconstructed by the hemi-pulp flap. The anterolateral thigh flap harvested from the left thigh transferred to the donor site defect of the hemi-pulp flap. Sixteen days after the first operation, another anterolateral thigh flap harvested from the left thigh transferred to the defect of the left middle finger. This operative procedure is very useful for cases requiring multi-flap transfer.
\end{abstract}

\section{Keywords}

Anterolateral Thigh Flap, Twice from the Ipsilateral Thigh, Perforator Flap

\section{Introduction}

Several cutaneous perforators arise from the descending branch of the lateral circumflex artery at the lateral thigh, and these perforators nourish the anterolateral thigh flap. Until now, harvesting of an anterolateral thigh

"Corresponding author.

How to cite this paper: Masami, S., Naohiro, K., Masayuki, O., Yosuke, T., Masamitsu, O. and Kazuki, U. (2016) A Case of Harvesting Anterolateral Thigh Flaps Twice from the Ipsilateral Thigh of a Single Patient in Separate Operations. Surgical Science, 7, 185-190. http://dx.doi.org/10.4236/ss.2016.74025 
flap with two skin paddles by utilizing two of these skin perforators has been reported [1], but harvesting of flap elevations from the ipsilateral thigh twice in the same patient in two operations has not been reported. We harvested anterolateral thigh flaps from the ipsilateral thigh of a single patient on two occasions using two of these perforators.

\section{Case Report}

A 44-year-old man suffered a degloving injury to the right thumb and left middle finger (Figure 1(a), Figure 1(b)). The reconstructive surgeries of his right thumb and his left middle finger were planned in separate operations.

In the first operation, a great toe hemi-pulp flap was transferred to the defect of the right thumb (Figure 2). Without delay, ananterolateral thigh flap was harvested from the left thigh and then transferred to the donor site defect of the hemi-pulp flap (Figure 3, Figure 4). Sixteen days after the initial surgery, another anterolateral thigh flap was harvested from the left thigh and transferred to the defect of the left middle finger (Figure 5, Figure 6). The overlap of the first flap and the second flap was avoided by preoperative examination of the perforators with a Doppler probe. The second thigh flap was elevated from a more distal site relative to that of the first operation (Figure 7). At the first operation, suprafascial dissection was carried out to identify the perforator; at the second operation, infrafascial dissection was carried out for subcutaneous adhesion. Both perforators of the first operation and the second operation had pass through in the vastus lateralis muscle. The fascia was preserved in the thigh in both operations. His final postoperative course was uneventful and all transferred tissues survived. One year postoperatively, the patientused the reconstructed fingers naturally and could walk normally or with weight-bearing (Figures 8(a)-(c)).

\section{Discussion}

Usually, a case requiring double free flap reconstruction is rare. In such cases, flaps are harvested from different donor sites of the same patient in each operation. There are several skin perforators arising from the descending branch of the lateral femoral circumflex artery [2]. It is possible to elevate a large flap and two skin paddle flaps by utilizing two of these skin perforators [1]. Until now, harvesting of an anterolateral thigh flap with two skin paddles has been reported [1], but harvesting of flap elevations from the ipsilateral thigh twice in the same patient in two operations has not been reported. The advantage of double free flap transplantations from the same site is low donor site morbidity. In other flaps, such as a deep inferior epigastric artery perforator flap [3], a thoracodorsal artery perforator flap [4], and a tensor fasciae latae perforator flap [5], the perforators are too closely located to each other to allow elevation of the two flaps. Moreover, a groin flap has one dominant perforator [6], so it can-not provide two flaps. Accordingly, the anterolateral thigh flap is the only perforator flap that can be

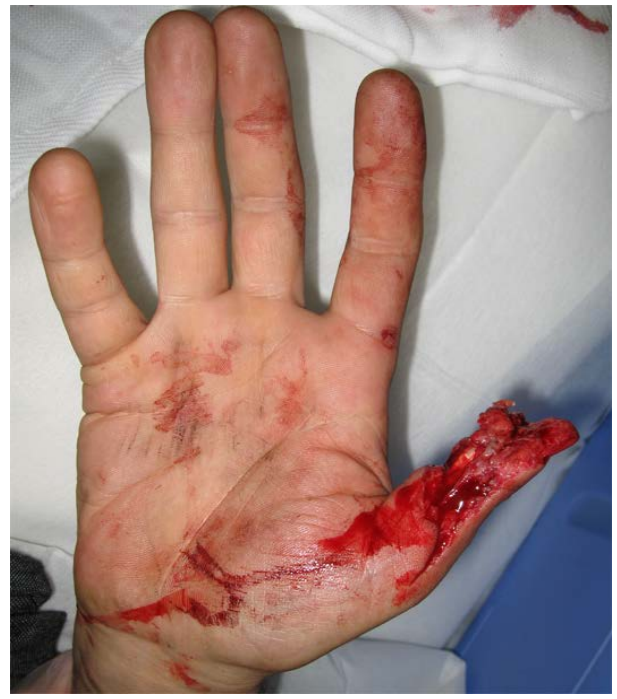

(a)

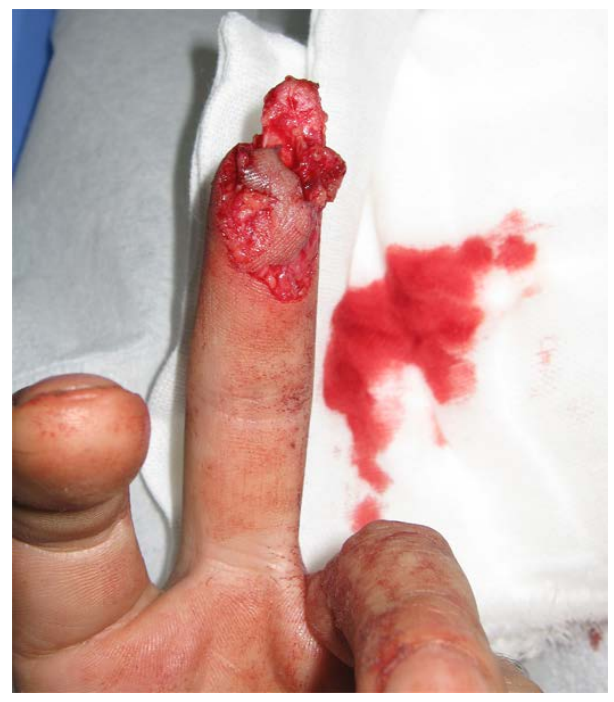

(b)

Figure 1. A 44-year-old man suffered a degloving injury to the right thumb and left middle finger. 


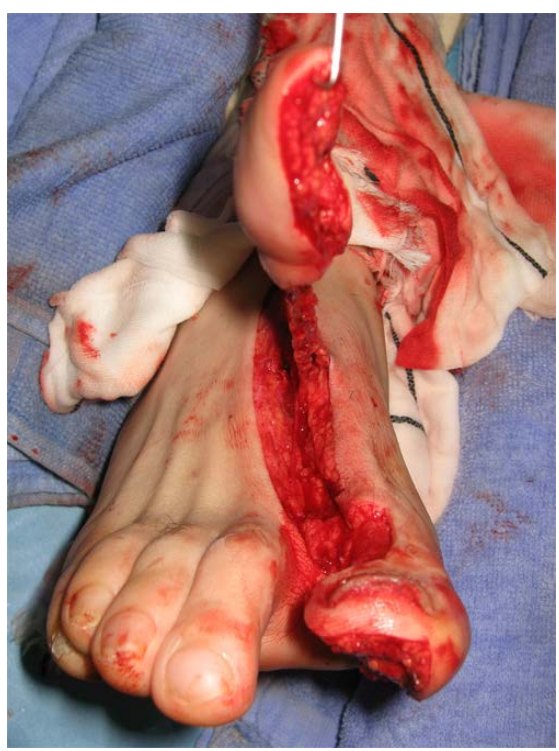

Figure 2. The right thumb was reconstructed by a hemi-pulp flap.

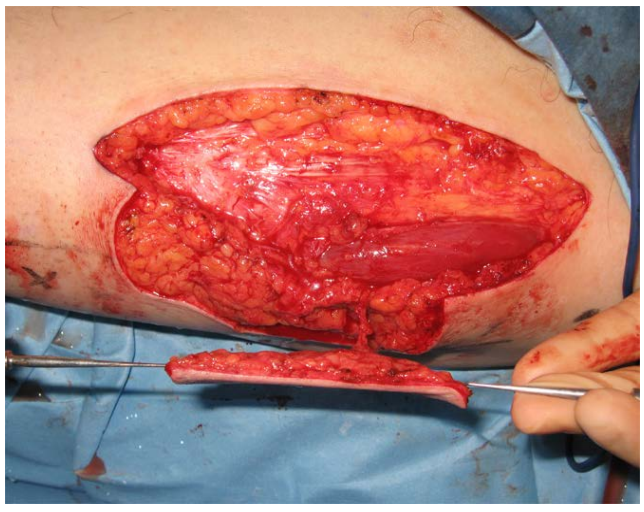

Figure 3. An anterolateral thigh flap was harvested from the left thigh.

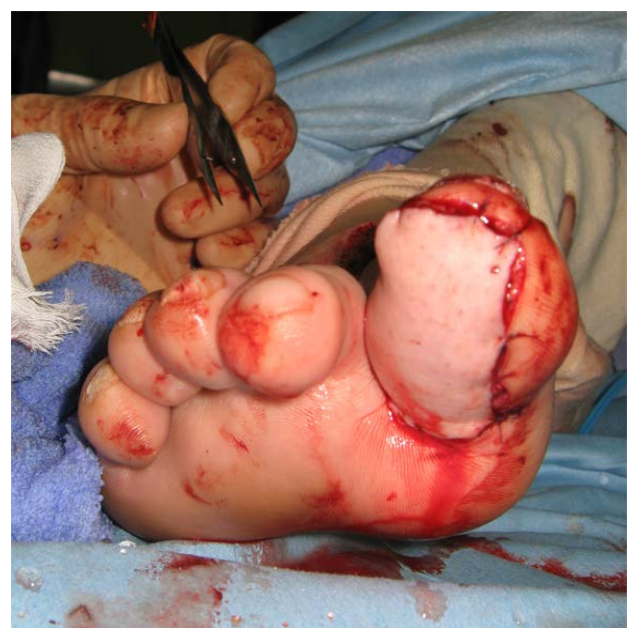

Figure 4. The thigh flap was transferred to the donor site defect of the hemi-pulp flap. 


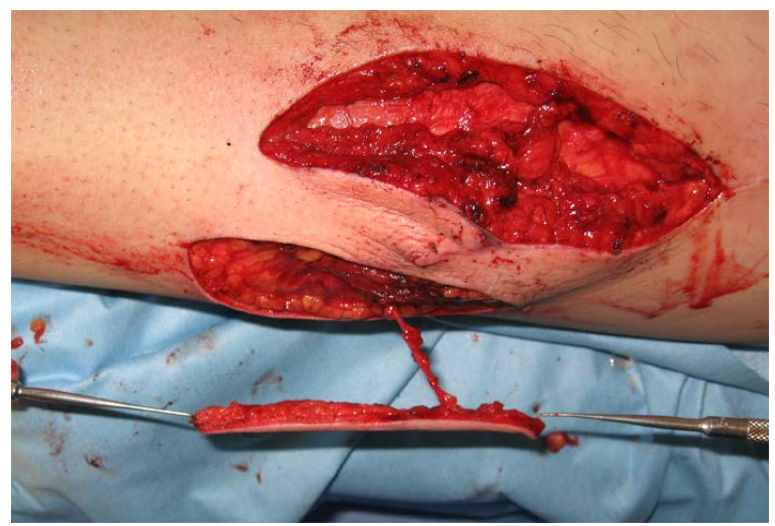

Figure 5. Sixteen days after the initial surgery, another anterolateral thigh flap was harvested from the left thigh.

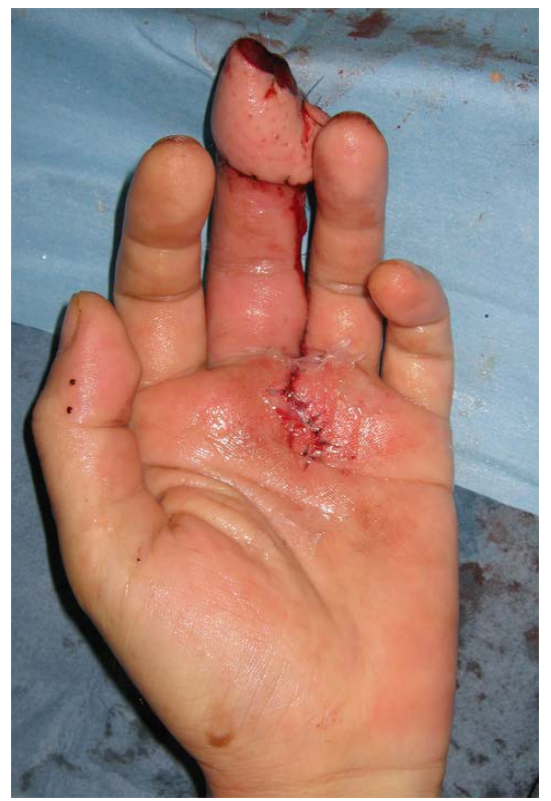

Figure 6. The second thigh flap was transferred to the defect of the left middle finger.

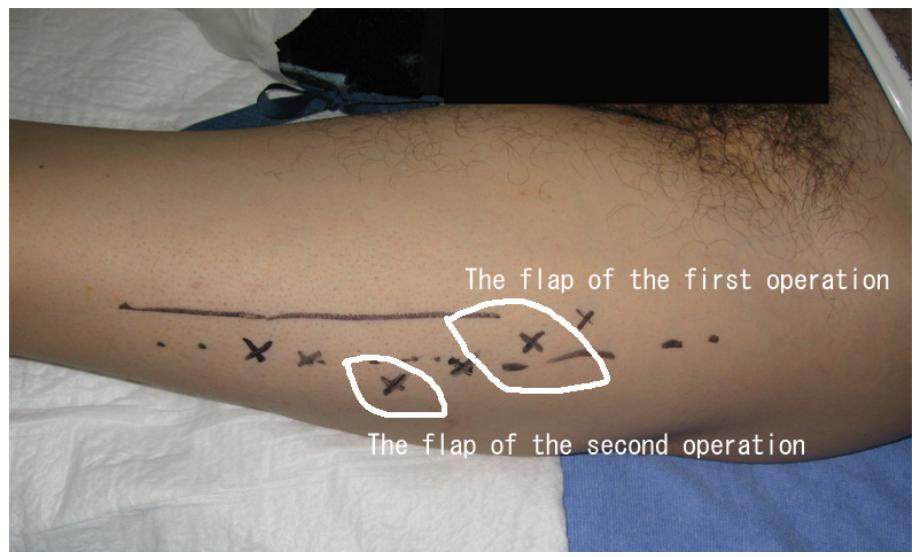

Figure 7. Preoperative examination of the perforators with a Doppler probe. 


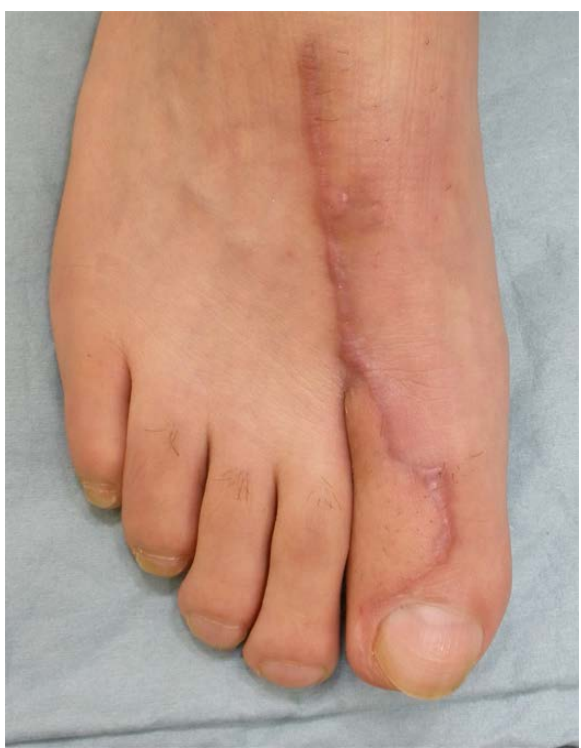

(a)

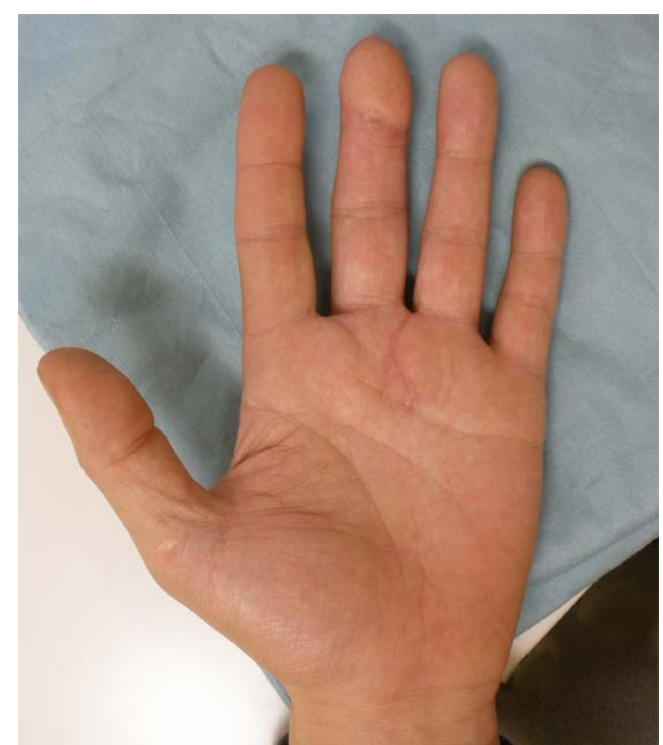

(b)

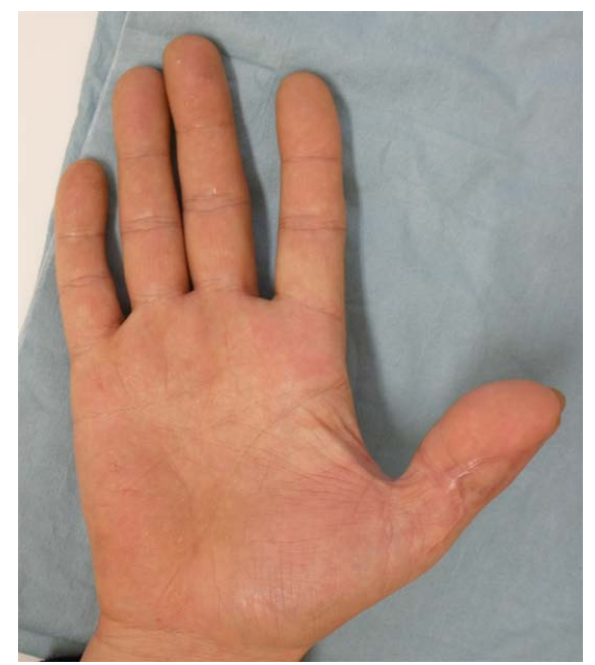

(c)

Figure 8. One year postoperatively, the patient used the reconstructed fingers naturally and could walk normally.

used for double flap harvesting from a single donor site. A problem that can be encountered at the second operation is that fascia-adipose layer scar adhesion caused by the initial surgery can impede dissection of the skin perforator. Dissection of a small perforator surrounded by the scar poses a high risk for damage of the perforator. However, at the first operation we located a perforator above the fascia, and at the second, we located another under the fascia of a scarless layer.

\section{Conclusion}

The anterolateral thigh flap is the only perforator flap that can be used for double flap harvesting from a single donor site. For patients requiring two flap reconstructions in separate operations, use of an anterolateral thigh flap allows limitation of donor site morbidity to a singlesite.

\section{References}

[1] Ao, M., Uno, K., Maeta, M., et al. (1999) De-Epithelialized Anterior (Anterolateral and Anteromedial) Thigh Flaps for 
Dead Space Filling and Contour Correction in Head and Neck Reconstruction. British Journal of Plastic Surgery, 52, 261-267. http://dx.doi.org/10.1054/bjps.1999.3107

[2] Koshima, I., Fukuda, H., Utunomiya, R., et al. (1989) The Anterolateral Thigh Flap: Variations in Its Vascular Pedicle. British Journal of Plastic Surgery, 42, 260-262. http://dx.doi.org/10.1016/0007-1226(89)90142-2

[3] Koshima, I. and Soeda, S. (1989) Inferior Epigastric Artery Skin Flap without Rectus Abdominis Muscle. British Journal of Plastic Surgery, 42, 645-648. http://dx.doi.org/10.1016/0007-1226(89)90075-1

[4] Kim, J.-T. (2003) Latissimus Dorsiperforator Flap. Clinics in Plastic Surgery, 30, 403-431. http://dx.doi.org/10.1016/S0094-1298(03)00041-5

[5] Koshima, I., Urushibara, K., Inagawa, K. and Moriguchi. T. (2000) Free Tensor Fasciae Latae Perforator Flap for the Reconstruction of Defects in the Extremities. Plastic and Reconstructive Surgery, 107, 1759-1765.

[6] Kimura, N. and Saitoh, M. (2006) Free Microdissected Thin Groin Flap Design with an Extended Vascular Pedicle. Plastic and Reconstructive Surgery, 117, 986-992. http://dx.doi.org/10.1097/01.prs.0000200618.85172.22 